\title{
Synthesis of amino-functionalized silica nanoparticles for preparation of new laboratory standards
}

\author{
Aitor Alvarez-Toral $^{1}$, Beatriz Fernández ${ }^{1 *}$, Julien Malherbe ${ }^{2}$, Fanny Claverie ${ }^{2}$, \\ Christophe Pecheyran ${ }^{2}$, Rosario Pereiro ${ }^{1}$
}

\begin{abstract}
${ }^{1}$ Department of Physical and Analytical Chemistry, Faculty of Chemistry; University of Oviedo; Julian Clavería, 833006 Oviedo, Spain

${ }^{2}$ Laboratoire de Chimie Analytique Bio-Inorganique et Environnement, IPREM UMR 5254, CNRS, Université de Pau et des Pays de l'Adour, 2 Avenue du Président Angot, 64053, Pau Cedex 9, France
\end{abstract}

*Author to whom correspondence should be addressed

Corresponding author: fernandezbeatriz@uniovi.es

Telephone: +34.985103524 


\begin{abstract}
Platinum group elements (PGEs) are particularly interesting analytes in different fields, including environmental samples as well as high cost materials that contain them, such as for example automotive catalysts. This type of solid samples could be analysed by laser ablation (LA) coupled to ICP-MS, which allow to significantly reducing the analysis time since the time-consuming processes for sample preparation are not required. There is a considerable demand of standards with high PGEs concentration for quantification purposes, which cannot be carried out easily using LA-ICP-MS because the available standards (i.e. NIST SRM 61x series) do not have such analytes in the same concentration range. In this paper, a new strategy is proposed for the synthesis of homogeneous laboratory standards with $\mathrm{Pt}, \mathrm{Pd}$ and $\mathrm{Rh}$ concentrations that range from $77 \mu \mathrm{g} / \mathrm{g}$ of Pd up to $2035 \mu \mathrm{g} / \mathrm{g}$ of $\mathrm{Rh}$. The proposed strategy is based on the synthesis of monodisperse amino-functionalized amorphous silica nanoparticles, which can retain metal ions. In addition to $\mathrm{Pt}, \mathrm{Pd}$ and $\mathrm{Rh}$, three lanthanides were also added to the nanoparticles (La, Ce, Nd). Sturdy pressed pellets can be made from the resulting nanopowder without the use of any binder. Elemental composition of standards made of nanoparticles was analysed by conventional nebulization ICP-MS and their homogeneity was successfully evaluated by LA-ICP-MS.
\end{abstract}

KEYWORDS: Laser ablation ICP-MS; Laboratory standards; Silica nanoparticles; Platinum group elements; Rare earth elements. 


\section{Introduction}

Laser ablation - inductively coupled plasma - mass spectrometry (LA-ICP-MS) is a well-known analytical technique which can be applied in a wide range of applications for solid sample analysis [1-3]. LA-ICP-MS has a remarkable position among the most used solid-state analysis techniques, thanks to its good lateral resolution and high sensitivity, allowing multi-elemental analysis at trace concentration levels $(<\mu \mathrm{g} / \mathrm{g})$ [4,5]. Its low sample removal [6] makes LA-ICP-MS a very attractive technique to analyse limited amount of samples. Furthermore, it can be easily employed for analysis of powdered samples [7] opposite to other techniques that present some limitations due to vacuum requirements (e.g. glow discharge sources or secondary ion mass spectrometry).

Although qualitative and semi-quantitative analysis is almost a routine in LA-ICPMS, calibration for quantitative analysis still remains an important challenge for a large variety of samples. Additionally, LA-ICP-MS applications are continuously increasing and, thus, the need of new standards for quantification purposes is becoming more and more important. In some cases, the available standards do not allow an adequate calibration strategy since the concentrations of the analytes in the samples are not in the range of the standards concentrations. In this sense, platinum group elements (PGEs) determination can be difficult since the most employed LA-ICP-MS standards (such as NIST SRM 61x series) do not have concentrations of PGEs higher than $4 \mu \mathrm{g} / \mathrm{g}[8,9]$. To overcome this limitation, several approaches can be investigated to make standards with higher PGEs concentrations, such as the synthesis of home-made matrix-matched standards using powdered materials with addition to a binder or another matrix $[10,11]$.

It would be interesting to search on the preparation of homogeneous standards which elemental concentrations are determined by the amount of the element added during the synthesis step. Until recently, the manufacturing of synthetic standards was carried out either by melting and flash cooling powdered materials or by chemical precipitation $[12,13]$. These methods allow the control of the concentrations of the elements in the resulting materials. However, both strategies have major drawbacks: the precipitation method provides materials whose homogeneity is restricted by the particle size distribution of the obtained powder, and the melting method cannot provide materials with high concentrations of volatile elements. Another approaches reported in the literature are based on the addition of a solution (natural abundance or isotopically- 
enriched isotopes) of the analytes to a powdered standard with the subsequent pelletization or fusion [14-16].

Lately, a different approach for inorganic solid standard synthesis was studied by using nanoparticles (NPs). NPs offer a high surface to volume ratio which helps to maximise the amount of metal elements that can be retained. NPs can be synthesized by either top-down (from macroscopic materials to NPs [17]) or bottom-up (from atoms to NPs [12]) strategies. In particular, Tabersky et al. [12] used a bottom-up approach based on a flame spray technique to synthesize nanopowders containing major elements (in the single wt\%-range) and rare earth elements and precious metals (between 300-1000 $\mu \mathrm{g} / \mathrm{g}$ ). The proposed strategy allows obtaining customized production of reference materials with a similar quality as reported for NIST glass. However, a large spatial variation of PGEs' concentration in the standards as well as contamination during production for some elements was reported. Both for top-down and bottom-up approaches, not only composition homogeneity is required but also a narrow dispersed particle size distribution to obtain standards of high analytical quality.

High purity $\mathrm{SiO}_{2}$ nanoparticles can be synthesized with different particle size and shape [18]. Taking into account that monodispersity is also a requirement to obtain appropriate standards, $\mathrm{SiO}_{2}$ is an interesting material because there are synthesis protocols that produce monodisperse silica nanoparticles [19-22]. Moreover, amorphous silica NPs can be easily functionalized to improve metal ions retention [23,24]. Thus, the synthesis of new laboratory standards for LA-ICP-MS analysis via aminofunctionalized amorphous silica nanoparticles is here proposed. Bearing in mind that standards with high concentrations of PGEs are still not available in the market (and they are of high interest for the analysis of technological samples as automotive catalysts), Rh, Pt and Pd have been selected as model analytes for PGEs. As the ideal standard should contain a wide range of analytes (to be applied for the simultaneous analysis of different elements), the addition of three lanthanides ( $\mathrm{La}, \mathrm{Ce}$ and $\mathrm{Nd}$ ) was also carried out. Furthermore, considering that an internal standard could be of interest for the development of a quantification method by LA-ICP-MS, Tl was also added to the NPs since it is one of the elements typically used for such purpose. Different batches of amino-functionalized amorphous silica NPs, with two ranges of elements concentrations, were synthesized and characterized by electron microscopy and LAICP-MS. 


\section{Experimental}

\subsection{Reagents and Materials}

The nanoparticles were synthesized and purified using the following reagents: TEOS (tetraethyl orthosilicate, 99.999\% trace metal basis, Aldrich), absolute ethanol (VWR Chemicals), 2.0 M ammonia solution in ethanol (Sigma-Aldrich), APTES (3triethoxysilylpropylamine, minimum 98\%, Sigma) and hexane (CHROMASOLV for HPLC, $\geq 95 \%$, Sigma Aldrich). Rh, Pd, Pt, La, Ce, Nd and Tl were added to the nanoparticles as a solution prepared from the following reagents, respectively: $\mathrm{RhCl}_{3} \cdot \mathrm{H}_{2} \mathrm{O}$ (99.99\%, Alfa Aesar), $\mathrm{Pd}\left(\mathrm{NO}_{3}\right)_{2} \cdot 2 \mathrm{H}_{2} \mathrm{O}$ ( 40\% Pd basis, Aldrich), $\mathrm{H}_{2} \mathrm{Cl}_{6} \mathrm{Pt}$ (8 wt.\% in $\mathrm{H}_{2} \mathrm{O}$, Aldrich), $\mathrm{LaCl}_{3}$ (99.999\% trace metals basis, Aldrich), $\mathrm{CeCl}_{3} \cdot 7 \mathrm{H}_{2} \mathrm{O}$ (99\% purity, Alfa Aesar), $\mathrm{Nd}\left(\mathrm{NO}_{3}\right)_{3} \cdot 6 \mathrm{H}_{2} \mathrm{O}$ (99.9\% trace metal basis, Aldrich) and $\mathrm{TINO}_{3}$ (99.999\% trace metals basis, Aldrich). The following element standards were used for quantification of digested samples by ICP-MS: Nd and Gd ICP standards, PlasmaCal, $1000 \mu \mathrm{g} / \mathrm{mL}$ in $4 \% \mathrm{HNO}_{3}$ from SCP Science; In, Rh, Pd, La, and Ce ICP Standards, Certipur®, $1000 \mathrm{mg} / \mathrm{L}$ each in 2-3\% $\mathrm{HNO}_{3}$ from Merck, and Pt ICP Standard, Certipur®, $1000 \mathrm{mg} / \mathrm{L}$ in $7 \% \mathrm{HCl}$ from Merck. For the purification of aminofunctionalized nanoparticles, Amicon Ultra-15 (MWCO $50 \mathrm{kDa}$, sample volume 15 $\mathrm{mL}$ ) filters from Millipore were used.

\subsection{Instrumentation}

The NPs were characterized by Scanning Electron Microscopy (SEM) with a JSM-6610LV instrument. Carbon paste was used to stick the nanoparticles on the SEM sample holder. Since the amorphous silica NPs are not electrically conductive, gold coating was applied to them before SEM measurements.

The digestion of the NPs was carried out in a Milestone ETHOS One Microwave Digestion System, from Microwave Laboratory Systems. ICP-MS measurements for quantitative analysis of the NPs by conventional nebulization were carried out with a 7500ce system (quadrupole ICP-MS) from Agilent Technologies. Experimental conditions selected for the measurements are summarised in Table 1.

The studies of homogeneity of the functionalized NPs were done by coupling a Nd:YAG LSX-213 laser ablation system from Teledyne Cetac Technologies to the quadrupole ICP-MS. Experimental conditions for LA-ICP-MS measurements are collected in Table 1 as well. The ICP-MS system is tuned with a multi-element tuning 
solution containing $1 \mathrm{ng} / \mathrm{g}$ of $\mathrm{Li}, \mathrm{Y}, \mathrm{Ce}$ and $\mathrm{Tl}$ for maximum sensitivity, low oxide formation $(<2 \%)$ and low double charged species $(<3 \%)$. The quadrupole ICP-MS was equipped with an octapole cell to remove polyatomic interferences for selected analytes $\left({ }^{29} \mathrm{Si}^{+}\right.$is measured for normalisation purposes, as $\mathrm{SiO}_{2}$ is the matrix of our NPs). In this study the collision cell was operated with a He gas flow of $3.9 \mathrm{~mL} / \mathrm{min}$. Additionally, LA-ICP-MS coupling was also optimised daily using a SRM NIST 612 glass standard for maximum sensitivity, low background intensity, and ${ }^{238} \mathrm{U} /{ }^{232} \mathrm{Th}$ signal ratio close to 1 (to ensure a low fractionation effect due to the ICP ionisation efficiency). ${ }^{248} \mathrm{ThO} /{ }^{232} \mathrm{Th}$ signal ratio was also measured for controlling oxide formation, being always below $2 \%$ at the selected conditions.

Table 1. Experimental conditions used for the ICP-MS analysis of the digested silica NPs and the LA-ICP-MS measurements of pressed pellets.

\begin{tabular}{|c|c|c|}
\hline Parameter & ICP-MS & LA-ICP-MS \\
\hline Sample gas flow (Ar) & $1100 \mathrm{~mL} / \mathrm{min}$ & $700 \mathrm{~mL} / \mathrm{min}$ \\
\hline Collision cell gas flow (He) & $3.9 \mathrm{~mL} / \mathrm{min}$ & $3.9 \mathrm{~mL} / \mathrm{min}$ \\
\hline Replicates per measurement & 10 & 1 ("time resolved analysis") \\
\hline Integration time & $\begin{array}{c}0.1 \mathrm{~s} \text { per point } \\
(0.3 \mathrm{~s} \text { per mass })\end{array}$ & $\begin{array}{c}0.1 \mathrm{~s} \\
\left(0.05 \mathrm{~s} \text { for }{ }^{29} \mathrm{Si}\right)\end{array}$ \\
\hline Laser energy & - & $5.6 \mathrm{~mJ}$ \\
\hline Laser repetition rate & - & $20 \mathrm{~Hz}$ \\
\hline Laser spot size & - & $200 \mu \mathrm{m}$ \\
\hline Laser scan rate & - & $10 \mu \mathrm{m} / \mathrm{s}$ \\
\hline He gas flow rate (ablation cell) & - & $800 \mathrm{~mL} / \mathrm{min}$ \\
\hline
\end{tabular}

\subsection{Experimental Procedures}

Synthesis of amorphous silica nanoparticles.- The first step for the preparation of the proposed laboratory standards is the synthesis of the amorphous silica NPs, which was carried out following a simple sol-gel process [22]. All the processes related to the synthesis of functionalized silica NPs were done in a fume hood. $348 \mathrm{~mL}$ of absolute ethanol was put in a clean glass bottle and $124 \mathrm{~mL}$ of the ammonia solution was added to the ethanol along with $8 \mathrm{~mL}$ of Milli-Q water. The mixture was stirred and the bottle 
was kept in water at a constant temperature of $25^{\circ} \mathrm{C}$ before adding the precursor. $20 \mathrm{~mL}$ of TEOS were added and the mixture was kept at $25{ }^{\circ} \mathrm{C}$ for at least $8 \mathrm{~h}$ under fast stirring. Once the reaction has finished, the NPs were purified by centrifugation at 5000 $\mathrm{g}$ for $1 \mathrm{~h}$ (an equivalent volume of hexane is added in the centrifugal tubes in order to precipitate the nanoparticles). The NPs were thoroughly purified by washing them twice with hexane. After each washing step, the centrifugation process was carried out and the supernatant hexane was discarded (the NPs are left in the bottom of the tube). After that, the centrifuge tubes were opened in order to get dry nanopowder (taking precautions to avoid dust deposition and any other contamination). Finally, the nanopowder was milled with an agate mortar in order to crush the possible clumps before functionalization.

Functionalization of silica nanoparticles.- The nanoparticles were functionalized by adding $450 \mu \mathrm{L}$ of APTES to $0.45 \mathrm{~g}$ of not functionalized amorphous silica NPs suspended in $30 \mathrm{~mL}$ of ethanol (95\%), using a glass vial and a magnetic stirrer. Just before the addition of APTES, the suspension of NPs was sonicated for 15 min to remove possible clumps and, thus, to maximize the surface available for functionalization. After sonication and subsequent addition of APTES, the mixture was stirred at room temperature for at least $8 \mathrm{~h}$. The purification process used for the nonfunctionalized NPs was carried once again for the amino-functionalized silica NPs (centrifugation and washing steps). These NPs were also dried at room temperature by leaving the nanopowder in the opened centrifuge tubes.

Addition of selected elements to silica NPs.- $\mathrm{Rh}, \mathrm{Pt}, \mathrm{La}, \mathrm{Ce}, \mathrm{Nd}$ and $\mathrm{Tl}$ salts were dissolved in Milli-Q water and individual solutions (10000 $\mu \mathrm{g} / \mathrm{g}$ metal concentration) were prepared, using sonication and slight heating if necessary. In the case of Pd, a solution with $10000 \mu \mathrm{g} / \mathrm{g}$ of metal could not be obtained due to a limited solubility of $\mathrm{Pd}\left(\mathrm{NO}_{3}\right)_{2} \cdot 2 \mathrm{H}_{2} \mathrm{O}$ reagent, and a solution of $1200 \mu \mathrm{g} / \mathrm{g}$ of Pd was employed. Solutions of the chloride salts ( $\mathrm{Rh}, \mathrm{Pt}, \mathrm{La}$ and $\mathrm{Ce}$ ) were mixed and a silver nitrate solution was added in order to precipitate chloride ions, which would cause precipitation of $\mathrm{Tl}$ (a $20 \%$ excess of $\mathrm{AgNO}_{3}$ was added to ensure complete precipitation). Then, the mixture was centrifuged (1000 g, $15 \mathrm{~min})$ and the supernatant was separated from the precipitate, which was washed with Milli-Q water twice in order to recover any metal (besides silver) that could be in the precipitate. 
$\mathrm{Pd}, \mathrm{Tl}$ and $\mathrm{Nd}$ solutions were afterwards mixed with the supernatant from the chloride precipitation (i.e. $\mathrm{Rh}, \mathrm{Pt}, \mathrm{La}$ and $\mathrm{Ce}$ ), getting a multi-element solution with all the selected analytes. pH control is critical in the proposed synthesis strategy and it was carefully controlled during the preparation of the final multi-element solution. The higher the $\mathrm{pH}$ the better for metal binding; however, the PGEs can precipitate at a certain $\mathrm{pH}$ value. $\mathrm{pH}$ of solutions around 2 was found to be the most appropriate for the synthesis. Thus, multi-element solution (batch A) was made to the final volume and ammonia was used to adjust to the optimum $\mathrm{pH}$ for the synthesis process $(\mathrm{pH} 2)$. Elemental concentrations of the metals in the multi-element solution are collected in Section 3.2. Batch B solution was produced by diluting 1:1 an aliquot of batch A.

The amino-functionalized silica NPs $(\sim 0.8 \mathrm{~g})$ and $10 \mathrm{~mL}$ of the solution with the metal elements ( $\mathrm{Rh}, \mathrm{Pd}, \mathrm{Pt}, \mathrm{La}, \mathrm{Ce}, \mathrm{Nd}$ and $\mathrm{Tl}$ ) were mixed and sonicated for $15 \mathrm{~min}$ in order to produce disaggregation of the NPs and, thus, to increase the specific surface. After sonication, the NPs were separated from the solution by ultrafiltration, using Amicon Ultra-15 filters at $5000 \mathrm{~g}$ for $1 \mathrm{~h}$. The remaining NPs were transferred then to a vial using absolute ethanol in order to thoroughly draw it from the filters. The resulting suspension of nanoparticles in ethanol was dried with constant stirring until complete dryness. The agate mortar was used to get a finely divided powder.

Preparation of pellets by compaction.- NPs standards with the elements of interest were used to obtain pressed pellets for their subsequent analysis by LA-ICPMS. A $5 \mathrm{~mm}$ diameter pellet die was used to press the nanopowder (applying 2 tons for 5 min). Figure 1 collects an image of a pressed pellet.

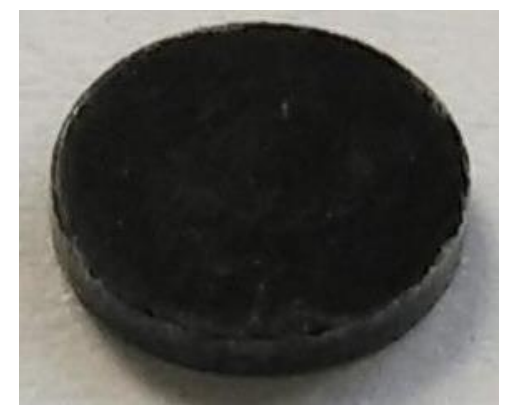

Figure 1. Photograph of a pressed pellet obtained from functionalized silica NPs. 
Microwave-assisted acid digestion.- In order to determine the analytes concentration in the synthesized laboratory standards, the amino-functionalized silica NPs were characterized by conventional nebulization ICP-MS after digestion using a 2step microwave-assisted acid digestion protocol [12]. In the first step, $10 \mathrm{~mL}$ of $\mathrm{HCl}, 3$ $\mathrm{mL}$ of $\mathrm{HNO}_{3}$ and $1 \mathrm{~mL}$ of $\mathrm{HF}$ were added to $50 \mathrm{mg}$ of NPs and the mixture was brought to a microwave with the following program: $10 \mathrm{~min}$ ramp from room temperature to $195^{\circ} \mathrm{C}$ and $30 \mathrm{~min}$ at a constant temperature of $195^{\circ} \mathrm{C}$ (max. power: $450 \mathrm{~W}$ ). In the second step, $10 \mathrm{~mL}$ of a saturated solution of boric acid was added. The microwave program in this second step consisted in a $10 \mathrm{~min}$ ramp from room temperature to 150 ${ }^{\circ} \mathrm{C}$ (max. power: $300 \mathrm{~W}$ ). After cooling to room temperature, the resulting acid solutions were diluted with $2 \% \mathrm{HNO}_{3}$. In and $\mathrm{Gd}$ were added during the dilution steps to be used as internal standards for quantitative ICP-MS analysis (external calibration).

\section{Results and Discussion}

\subsection{Synthesis and Microscopy Characterisation of Amorphous Silica NPs}

Large size amorphous silica NPs (greater than $100 \mathrm{~nm}$ in diameter) are easier to synthesize compared to small silica NPs, because the amount of water required for the synthesis is not so restricted (ammonia can be added as an aqueous solution). However, our experiments showed that pressed pellets made from such large size NPs are not sturdy enough for manipulation as they tend to disaggregate. Since pellets have to be prepared for the analysis of powdered samples by LA-ICP-MS, robust and compact pellets are required from the synthesized standards. Thus, it was aimed at synthesizing amorphous silica NPs smaller than $50 \mathrm{~nm}$, which requires the addition of ammonia as a solution in ethanol.

Figure 2a collects a SEM image obtained for the synthesized silica NPs. Pellets made from these smaller NPs were found to be more robust in comparison with larger NPs of $200 \mathrm{~nm}$ (results not shown), which is consistent with previous reported studies [11]. SEM images show agglomerates of small NPs with diameters close to $50 \mathrm{~nm}$. It should be stated that due to the drying process as well as to the gold coating process of the samples (both required for SEM measurements), agglomerates were observed instead of homogeneous single NPs. However, the shape of the aggregates obtained indicates that they are formed by spherical NPs with diameters around $50 \mathrm{~nm}$. These NPs with diameters were found to be appropriate for preparing pressed pellets suitable 
for LA-ICP-MS analysis. It has to be highlighted that the employment of small nanoparticles avoids the use of binders, thus eliminating the dilution of the analytes in the binder and eliminating a potential contamination source.

Next, the silica NPs were functionalized and subsequently characterized by SEM in order to make sure that the functionalization process does not produce measurable alterations in the size or shape of the original NPs. In Figure $2 b$ it can be observed the SEM image obtained after functionalization of silica NPs. As for the nonfunctionalized, the functionalized NPs have a homogeneous size, with a diameter close to $50 \mathrm{~nm}$. Finally, the amino-functionalized amorphous silica NPs were added to vials that contained the solution with the model elements ( $\mathrm{Rh}, \mathrm{Pd}, \mathrm{Pt}, \mathrm{La}, \mathrm{Ce}$ and $\mathrm{Nd})$. The amino-functionalized amorphous silica NPs were added to vials that contained two solutions of the elements with different concentration: batch A was prepared by using the original solution and batch B was prepared by using a diluted solution. Figure 2c collects a SEM image obtained for the amino-functionalized amorphous silica NPs with the elements incorporated (batch A with higher concentrations). As can be observed, the addition of the analytes neither affects the size nor the shape of the nanoparticles, as no significant differences were observed between the SEM images of the metal-free NPs (Figures $2 \mathrm{a}$ and $2 \mathrm{~b}$ ) and the NPs after the metal addition (Figure 2c). 
a)

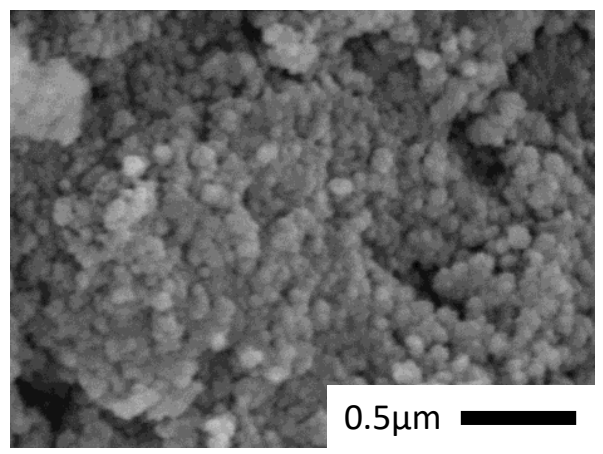

b)

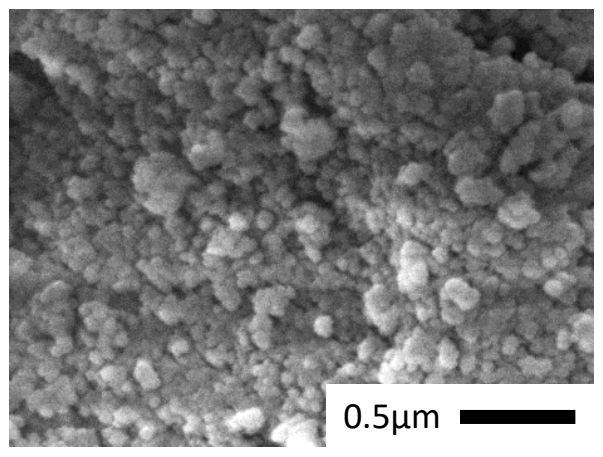

c)

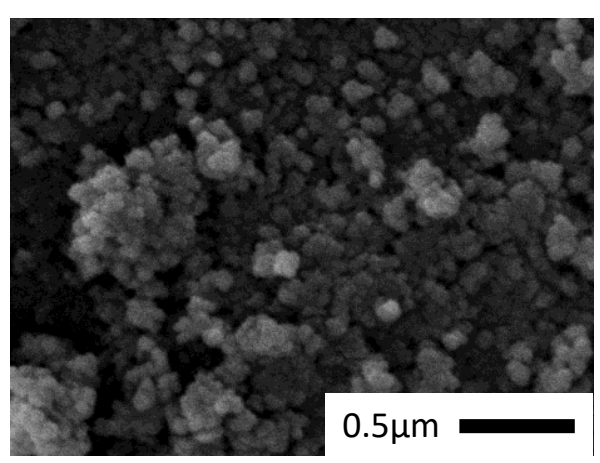

Figure 2. SEM images obtained from synthesized amorphous silica nanoparticles. a) Non-functionalized NPs; b) Amino-functionalized NPs before metal addition; c) Amino-functionalized silica nanoparticles after metal addition $(\mathrm{Pt}, \mathrm{Pd}, \mathrm{Rh}, \mathrm{La}, \mathrm{Ce}$ and $\mathrm{Nd})$.

\subsection{Measurement of Elemental Concentrations in the Silica NPs}

The elemental concentrations of the analytes in batches A and B solutions were experimentally measured by conventional nebulization ICP-MS in order to get information about the binding yields (defined as the relationship between the concentration of the added metals in the original solution and the final concentration of each metal in the synthesized amino-functionalized amorphous silica nanoparticles). Experimental results showed Pt concentrations in solutions significantly lower than expected values (up to one order of magnitude lower), suggesting a co-precipitation of such metal in the solution preparation step. Pd and $\mathrm{Nd}$ showed concentrations around $250 \mu \mathrm{g} / \mathrm{g}$, and $\mathrm{Rh}, \mathrm{La}$ and Ce in the range of $175-200 \mu \mathrm{g} / \mathrm{g}$.

The two batches (A and B) of metal enriched NPs were digested using the microwave-assisted digestion protocol described in the Experimental Procedures 
section. Next, they were submitted to quantification by conventional nebulization ICPMS using In and Gd as internal standards. The concentrations (expressed as micrograms of each element to grams of solid sample) obtained for $\mathrm{Pt}, \mathrm{Pd}, \mathrm{Rh}, \mathrm{La}, \mathrm{Ce}$ and $\mathrm{Nd}$ in the two batches are collected in Table 2. As expected, it was observed that the higher the concentration in the metals solution, the higher the concentration in the resulting nanopowder.

Table 2. Concentration $(\mu \mathrm{g} / \mathrm{g})$ of the added metal elements in two batches of amino-functionalized silica NPs measured by conventional nebulization ICP-MS.

\begin{tabular}{|c|c|c|}
\hline Element & Batch A $(\mu \mathbf{g} / \mathbf{g})$ & Batch B $(\mu \mathbf{g} / \mathbf{g})$ \\
\hline Rh & $2035 \pm 53$ & $1193 \pm 31$ \\
\hline Pd & $147 \pm 4$ & $77 \pm 2$ \\
\hline Pt & $204 \pm 5$ & $104 \pm 2$ \\
\hline La & $326 \pm 22$ & $214 \pm 19$ \\
\hline Ce & $739 \pm 51$ & $468 \pm 42$ \\
\hline Nd & $1336 \pm 92$ & $839 \pm 80$ \\
\hline
\end{tabular}

Experimental results clearly indicate that the different model analytes vary in their behaviour during the attachment to the silica NPs. Binding yields have been estimated for all the studied analytes and they can be classified in two groups: (i) $\mathrm{Rh}, \mathrm{Pd}$ and Pt (PGEs), with a high metal binding yield (45-95\%) and concentrations that are heavily dependent from the concentration in the standard solution; and (ii) lanthanides ( $\mathrm{La}, \mathrm{Ce}$ and $\mathrm{Nd}$ ), which have a lower metal binding yield (20-45\%) and whose concentration are less influenced by their concentration in the solution.

This behaviour can be attributed to the different chemical properties of the selected model analytes, in which the affinity of the amino group for the analytes seem to be crucial. The high binding yield of the PGEs can be attributed to the high affinity of these metals for the N-donor ligands, forming covalent metal-nitrogen bonds, as in other well-known PGE complexes [25] (including some used as catalysts [26]). On the other 
hand, the trivalent lanthanide cations ( $\mathrm{La}, \mathrm{Ce}$ and $\mathrm{Nd}$ ) form weaker, less covalent bonds with ligands, and as hard acid ions, they are expected to have affinity for hard bases such amino-groups. Metal-nitrogen bond takes place in lanthanide complexes with Ndonor ligands [27]. However these trivalent cations have also a high affinity to O-donor ligands, which means that interactions with monodentate $\mathrm{N}$-donor ligands in aqueous solutions are difficult due to water molecules from the coordination sphere are difficult to displace. As a result, lanthanide cations are retained with a considerable lower binding yield compared to PGE cations.

Although it is difficult to precisely predict the final concentration of the metals in the NPs standard, it can be easily performed by digestion of the nanopowders and the subsequent analysis by ICP-MS. Furthermore, it should be highlighted that using the proposed synthesis method, the concentrations of $\mathrm{Pt}$ and $\mathrm{Pd}$ in the amino-functionalized silica NPs were two orders of magnitude higher than the highest concentrations of the same analytes in the NIST SRM 61x series reference materials (Rh concentration was found to be three orders of magnitude higher).

\subsection{Study of the Laboratory Standards Homogeneity by LA-ICP-MS}

Pressed pellets from functionalized silica NPs were analysed by LA-ICP-MS. The ablation strategy selected for the analysis consisted on 5 ablation lines $(2.0 \mathrm{~mm}$ long using a $200 \mu \mathrm{m}$ laser beam diameter) per pellet with a $1 \mathrm{~mm}$ separation between lines. Experimental conditions employed for LA-ICP-MS analysis are summarised in Table 1. Figure 3 collects the ablation profiles obtained for the analysis of the NPs standard from batch B (Figure 3a) and batch A (Figure 3b). As can be observed, without taking into account the first and last seconds of the analysis $(\sim 25 \mathrm{~s})$ as it is normally done in laser ablation, the signals of all the elements were stable and no spikes were found on them, indicating a homogeneous distribution of the elements in the synthesized standards. Small signal variations observed in the profile seem to affect all the elements in the same order, suggesting that they were not caused by the presence of zones with different analytes concentration in the standard. 
a)

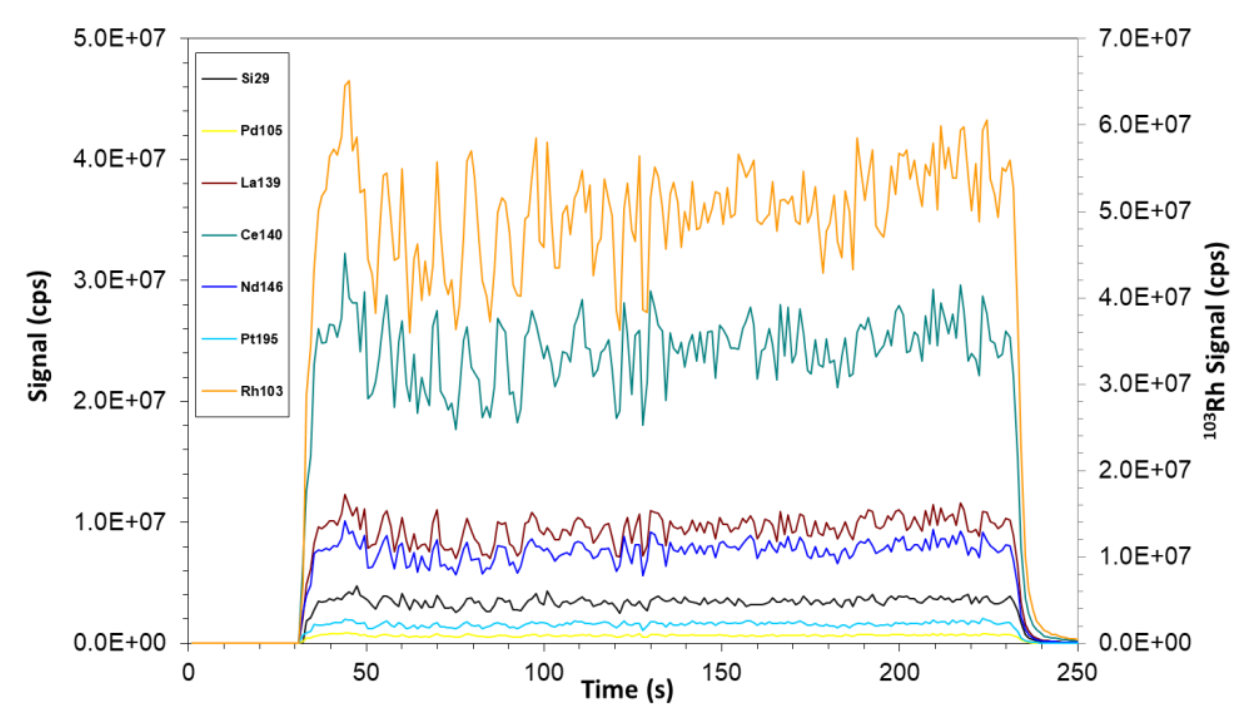

b)

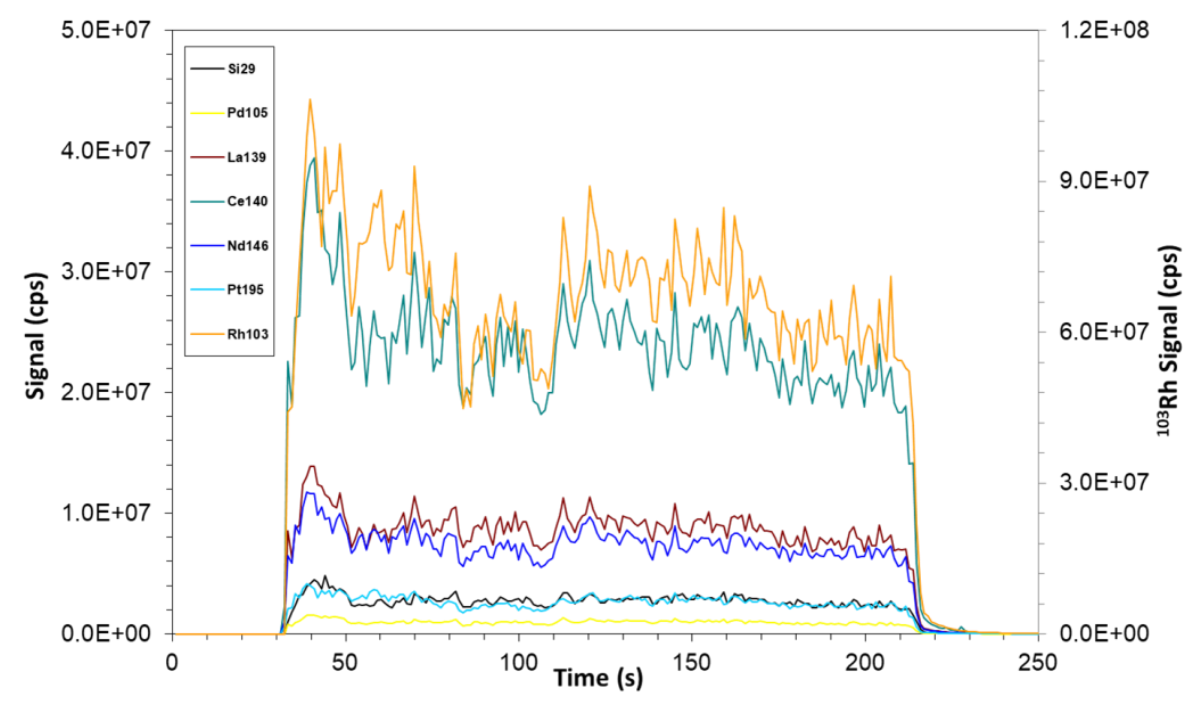

Figure 3. Ablation profiles obtained by LA-ICP-MS for the analysis of pressed pellets of functionalized silica NPs. a) Batch B, and b) Batch A.

Additionally, Figure 4 shows the profiles obtained for ${ }^{103} \mathrm{Rh}^{+},{ }^{195} \mathrm{Pt}^{+}$and ${ }^{105} \mathrm{Pd}^{+}$ signals normalised with ${ }^{29} \mathrm{Si}^{+}$signal (matrix of the standard) for pressed pellets from batch $\mathrm{B}$ and batch A (Figures $4 \mathrm{a}$ and $4 \mathrm{~b}$, respectively). Again, no significant signal variation was observed for PGEs elements and absence of spikes can be clearly 
observed in the profiles, suggesting in both cases the homogeneous distribution of the metals in the amino-functionalized silica NPs.

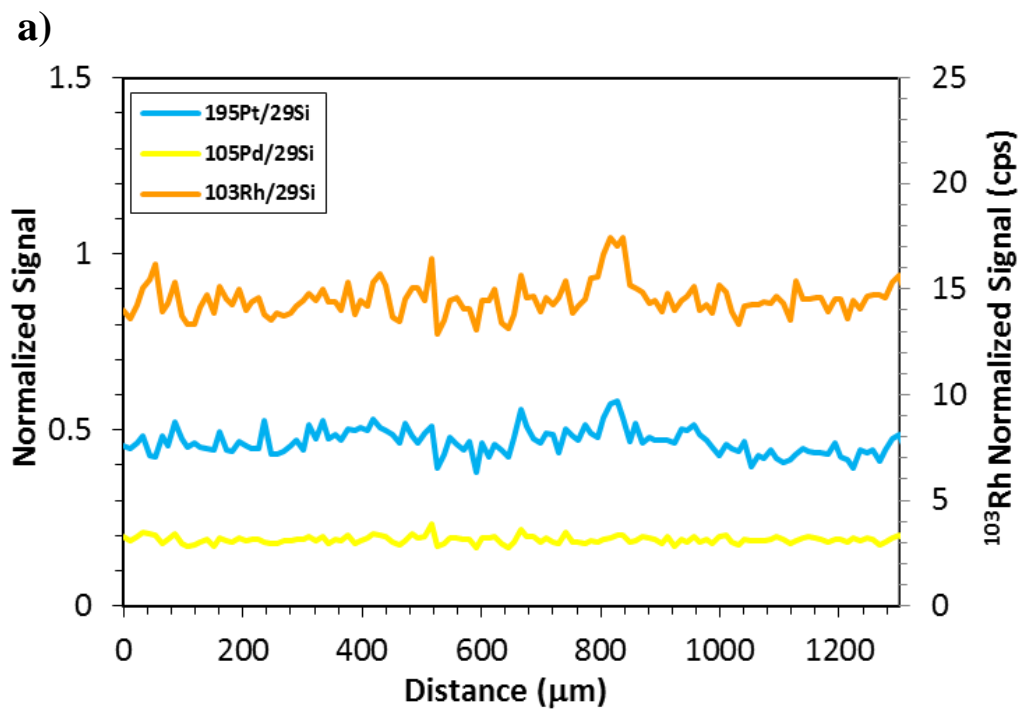

b)

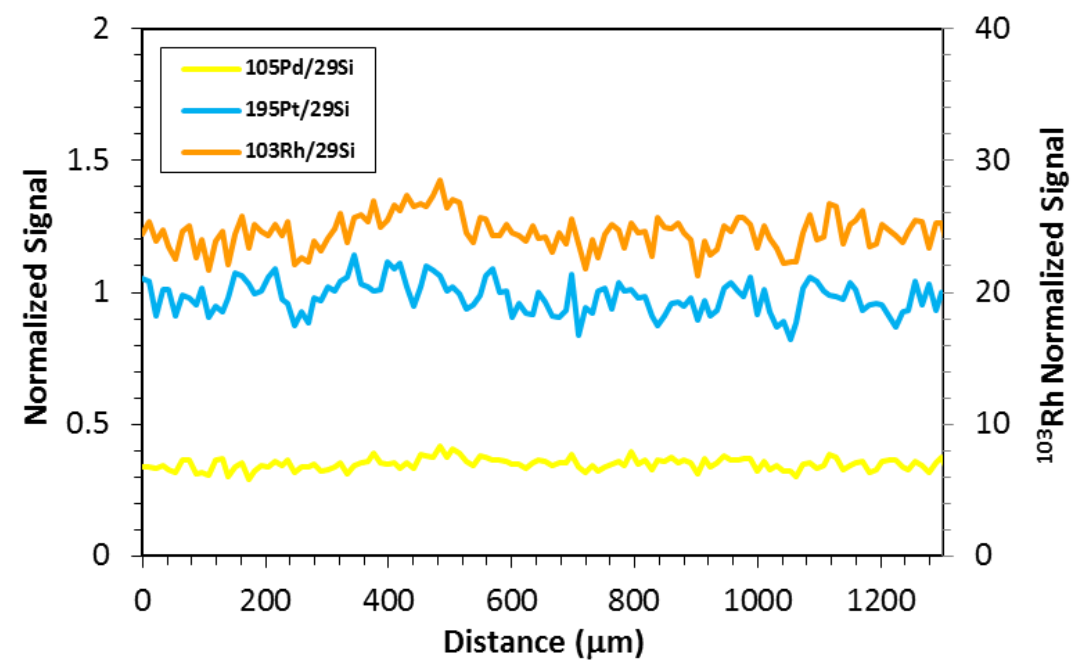

Figure 4. Variations of elemental responses of $\mathrm{Pd}, \mathrm{Pt}$ and $\mathrm{Rh}$ relative to $\mathrm{Si}$ along a line scan across a standard pellet ablated. a) Batch B, and b) Batch A.

Homogeneity of the standards was evaluated by LA-ICP-MS analysis by calculating the temporal relative standard deviation (tRSD), which is the relative standard deviation of each analyte signal (after spectral skew correction and normalization using ${ }^{29} \mathrm{Si}^{+}$signal) during the time interval of a laser ablation line (the tRSD values corresponds to a length of $1.5 \mathrm{~mm}$ since the first seconds of the LA profile were discarded). Furthermore, intra-pellet homogeneity was evaluated using the relative 
standard deviation (RSD) of the average signals from 5 ablation lines on the same pellet. Finally, the reproducibility of the amino-functionalized silica NPs synthesis process (denoted as the inter-pellet RSD) was studied by the analysis of different pellets from three independent synthesis: (i) 5 ablation lines were performed by LA-ICP-MS for each NPs pressed pellet; (ii) The average net signal intensity (cps) for all the elements in each line was calculated; (ii) As five ablation lines were done in each pellet, next the average net signal intensity was calculated for each pellet taking into account the average values of the five individual ablation lines; (iv) Finally, inter-pellet RSD was calculated as the relative standard deviation (RSD) using the average values of each pellet.

Table 3 collects the numerical values obtained for tRSD, intra- and inter-pellet homogeneity for the functionalized silica NPs from batch B (batch A was found to show similar values). tRSD values below $7.3 \%$ were obtained for the selected model analytes. On the other hand, both intra- and inter-pellet homogeneity were found to be in the range between 3.5\% and 7.6\% (except Pt that showed inter-pellets RSDs of 9.2\%) for the batch of elements with lower concentrations.

Table 3. Temporal relative standard deviation and intra- and inter-pellet relative standard deviation values obtained by LA-ICP-MS for the analysis of pressed pellets of amino-functionalized silica NPs (batch B).

\begin{tabular}{|c|c|c|c|}
\hline Element & tRSD (\%) & Intra-pellet (\%) & Inter-pellet (\%) \\
\hline $\mathbf{R h}$ & 6.1 & 5.4 & 4.2 \\
\hline $\mathbf{P d}$ & 6.3 & 3.5 & 7.0 \\
\hline $\mathbf{P t}$ & 7.2 & 5.8 & 9.2 \\
\hline $\mathbf{L a}$ & 7.1 & 7.6 & 7.2 \\
\hline $\mathbf{C e}$ & 7.3 & 5.0 & 6.7 \\
\hline $\mathbf{N d}$ & 6.8 & 5.9 & 5.8 \\
\hline
\end{tabular}

It can be stated that although inter-pellet homogeneity (that allowed evaluation of reproducibility between different syntheses of amino functionalized NPs) showed higher RSDs compared to intra-pellet homogeneity, RSD values below $10 \%$ were always found. Therefore, experimental results confirm that amino-functionalized silica 
NP standards containing six different elements (Pt, Pd, Rh, La, Ce and Nd) can be easily synthesized with an appropriate homogeneity to be used for quantification purposes using LA-ICP-MS.

\section{Conclusions}

The method proposed in the present work for the preparation of new laboratory standards for LA-ICP-MS analysis is based on the synthesis of amino-functionalized silica NPs (lower than $50 \mathrm{~nm}$ ). The method suits with the ideal preparation process for the synthesis of laboratory standards: is based on easy steps, it is low cost, and the resulting product material is homogeneous and able to contain several different elements in a large interval of concentrations.

Six elements were selected as model analytes: Pt, Pd, Rh (PGEs) and La, Ce, Nd (lanthanides). The proposed synthesis strategy was found to produce NPs whose pressed pellets are robust and homogeneous to be used for LA-ICP-MS analysis: pellets made from these metal-enriched nanoparticles are sturdy enough for an easy manipulation as they not tend to disaggregate like earlier proposed standard materials of nanopowders with higher nanoparticle size.

Different concentration levels for the metals were successfully investigated. However, further studies should be performed to be able to tune desirable concentration of the metals in the standard. In this sense, the addition of less number of elements per standard could possibly help to increase concentration level of the metals. In any case, concentration of model elements (particularly Pd, Pt and Rh) obtained in the standards was much higher (up to 3 orders of magnitude) compared to those found in the NIST SRM 61x series reference materials (generally employed for quantification purposes by LA-ICP-MS). The promising results obtained call for further investigations to apply the synthesized NPs standards for the analysis of powdered samples by LA-ICP-MS (for example, there is a high interest for quality control analysis of automotive catalysts where PGEs are extremely important).

\section{Acknowledgements}

The authors are grateful for the financial support from the "Plan de Ciencia, Tecnología e Innovación" (PCTI) of Gobierno del Principado de Asturias, European FEDER co-financing, and FICYT managing institution, through the project 
GRUPIN14-092. Beatriz Fernandez acknowledges her research contract RYC-201414985 to the Spanish Ministry of Economy and Competitiveness through the Ramón y Cajal Program.

\section{References}

[1] S.F. Durrant, N.I. Ward, Recent biological and environmental applications of laser ablation inductively coupled plasma mass spectrometry (LA-ICP-MS), J. Anal. At. Spectrom. 20 (2005) 821829.

[2] D. Pozebon, G.L Scheffler, V.L Dressler, M.A Goncalvez, Review of the applications of laser ablation inductively coupled plasma mass spectrometry (LA-ICP-MS) to the analysis of biological samples, J. Anal. At. Spectrom. 29 (2014) 2204-2228.

[3] A. Limbeck, P. Galler, M. Bonta, G. Bauer, W. Nischkauer, F. Vanhaecke, Recent advances in quantitative LA-ICP-MS analysis: challenges and solutions in the life sciences and environmental chemistry, Anal. Bioanal. Chem. 407 (2015) 6593-6617.

[4] J. Koch, D. Günther, "Review of the state-of-the-art of laser ablation inductively coupled plasma mass spectrometry", Appl. Spectrosc. 65 (2011) 155A-162A.

[5] J. Pisonero, B. Fernández, D. Günther, "Critical revision of GD-MS, LA-ICP-MS and SIMS as inorganic mass spectrometric techniques for direct solid analysis", J. Anal. At. Spectrom. 24 (2009) 1145-1160.

[6] T. Trejos, J.R. Almirall, Sampling strategies for the analysis of glass fragments by LA-ICP-MS

Part I. Micro-homogeneity study of glass and its application to the interpretation of forensic evidence, Talanta 67 (2005) 388-395.

[7] S.G. Boulyga, M. Tibi, K.G. Heumann; Application of isotope-dilution laser ablation ICP-MS for direct determination of $\mathrm{Pu}$ concentrations in soils at pg g-1 levels, Anal. Bioanal. Chem. 378 (2004) 342-347.

[8] M. Shaheen, J.E. Gagnon, Z. Yang, B.J. Fryer, Evaluation of the analytical performance of femtosecond laser ablation inductively coupled plasma mass spectrometry at $785 \mathrm{~nm}$ with glass reference materials, J. Anal. At. Spectrom., 23 (2008) 1610-1621.

[9] http://georem.mpch-mainz.gwdg.de/

[10] M. Resano, E. García-Ruiz, K.S. McIntosh, F. Vanhaecke, Laser ablation-inductively coupled plasma-dynamic reaction cell-mass spectrometry for the determination of platinum group metals and gold in NiS buttons obtained by fire assay of platiniferous ores, J. Anal. At. Spectrom. 23 (2008) 1599-1609.

[11] J. Hirata, T. Takahashi, M. Tanaka; Determination method of multi elements in ferromanganese samples by LA-ICP-MS, Anal. Sci. 29 (2013) 29, 151-155. 
[12] D. Tabersky, N.A. Luechinger, M. Rossier, E. Reuser, K. Hametner, B. Aeschlimann, D.A. Frick, S.C. Halim, J. Thompson, L. Danyushevsky, D. Günther, Development and characterization of custom-engineered and compacted nanoparticles as calibration materials for quantification using LAICP-MS, J. Anal. At. Spectrom. 29 (2014) 955-962.

[13] S. A. Wilson, W. I. Ridley, A. E. Koenig, Development of sulfide calibration standards for the laser ablation inductively-coupled plasma mass spectrometry technique, J. Anal. At. Spectrom. 17 (2002) 406-409.

[14] S.F. Boulyga, K.G. Heumann, Direct determination of platinum group elements and their distributions in geological and environmental samples at the ng g-1 level using LA-ICP-IDMS, Anal. Bioanal. Chem. 383 (2005) 442-447.

[15] F. Claverie, J. Malherbe, N. Bier, J.L. Molloy, S.E. Long, Standard addition method for laser ablation ICPMS using a spinning platform, Anal. Chem. 85 (2013) 3584-3591.

[16] B. Fernández, P. Rodríguez-González, J.I. García Alonso, J. Malherbe, S. García-Fonseca, R. Pereiro, A. Sanz-Medel; On-line double isotope dilution laser ablation inductively coupled plasma mass spectrometry for the quantitative analysis of solid materials, Anal. Chim. Acta 851 (2014) 6471.

[17] D. Garbe-Schönberg, S. Müller, Nano-particulate pressed powder tablets for LA-ICP-MS, J. Anal. At. Spectrom. 29 (2014) 990-1000.

[18] A. Liberman, N. Mendez, W.C. Trogler, A.C. Kummel, Synthesis and surface functionalization of silica nanoparticles for nanomedicine, Surface Science Reports 69 (2014) 132-158.

[19] S.K. Park, K.D. Kim, H.T. Kim, Preparation of silica nanoparticles: determination of the optimal synthesis conditions for small and uniform particles, Colloids Surf. A Physicochem. Eng. Asp. 197 (2002) 7-17.

[20] L.M. Rossi, L. Shi, F.H Quina, Z. Rosenzweig; Stöber synthesis of monodispersed luminescent silica nanoparticles for bioanalytical assays, Langmuir 21 (2005) 4277-4280.

[21] K. Suzuki, S. Sato, M. Fujita, Template synthesis of precisely monodisperse silica nanoparticles within self-assembled organometallic spheres, Nat. Chem. 2 (2010) 25-29.

[22] G.H. Bogush, M.A. Tracy, C.F. Zukoski, Preparation of monodisperse silica particles: control of size and mass fraction, J. Non-Cryst. Solids 104 (1988) 95-106.

[23] J. Liang, Z. Xue, J. Xu, H. Zhang, W. Yang, Highly efficient incorporation of amino-reactive dyes into silica particles by a multi-step approach, Colloids Surf. A Physicochem. Eng. Asp. 426 (2013) 33-38.

[24] Y. Zhao, B.G. Trewyn, I.I. Slowing, V.S.-Y. Lin, Mesoporous silica nanoparticle-based double drug delivery system for glucose-responsive controlled release of insulin and cyclic AMP, J. Am. Chem. Soc. 131 (2009) 8398-8400. 
[25] D. W. James, M J. Nolan, Vibrational spectra of substituted platinum (IV) amine complexes. II: cis and trans isomers of $\left[\mathrm{Pt}\left(\mathrm{NH}_{3}\right)_{4} \mathrm{X}_{2}\right]^{2+}$ and $\left[\mathrm{Pt}\left(\mathrm{NH}_{3}\right)_{2} \mathrm{X}_{4}\right]$ where $\mathrm{X}=\mathrm{Cl}, \mathrm{Br}$ and I. J. Raman Spectrosc. 1 (1973) 271-284.

[26] A. Tairai, P. K. Bhattacharyya, R. Kar, P. Das. Primary amine-based palladium (II) complexes as catalysts for Suzuki-Miyaura reaction: experimental and theoretical investigations on the effects of substituents on nitrogen atom. Indian J. Chemistry 51A (2012) 1545-1552.

[27] L. R. Melby, N. J. Rose, E. Abramson, J. C. Caris. Synthesis and fluorescence of some trivalent lanthanide complexes. J. Am. Chem. Soc. 86 (1964) 5117-5125. 